\title{
PREHOSPITAL CARE
}

\section{Recognition of death and termination of cardiac resuscitation attempts by UK ambulance personnel}

\author{
A S Lockey
}

Emerg Med J 2002;19:345-347

\begin{abstract}
A S Lockey, Accident and Emergency Department,

York District Hospital, York, UK

Correspondence to:

Dr A S Lockey, Accident and Emergency

Department, Calderdale Royal Hospital,

Salterhebble, Halifax,

Yorkshire, UK;

alockey@totalise.co.uk

Accepted for publication 9 November 2001
\end{abstract}

Objectives: To identify whether the practice of the UK ambulance trusts comply with national recommendations with respect to when ambulance personnel are allowed to recognise death and/or terminate resuscitation attempts in the adult, normothermic, non-traumatic cardiac arrest.

Methods: Questionnaire study of 39 ambulance trusts.

Results: At the time of the study (summer 2000), 23 trusts operated separate policies for recognition of death and termination of resuscitation, two had policies for recognition of death alone, two had policies for termination of resuscitation alone, five operated a policy purely for termination of resuscitation attempts after a limited period of CPR, and seven had no protocols other than "the presence of rigor mortis, postmortem staining or injuries incompatible with life". Only eight trusts conformed to the protocols for both recognition of death and termination of resuscitation attempts recommended by the Joint Royal Colleges Ambulance Liaison Committee (JRCALC).

Conclusion: The JRCALC has proposed guidelines for recognition of death and terminating resuscitation attempts in the adult normothermic non-traumatic cardiac arrest. Despite this, there was still considerable variance in the practice of the UK ambulance trusts.
I has been traditional in the United Kingdom (UK) for ambulance personnel to commence resuscitative measures on most normothermic adult cardiac arrest victims. The exceptions have been those patients who have rigor mortis, postmortem staining, or injuries incompatible with life. The Joint Royal Colleges Ambulance Liaison Committee (JRCALC) published guidelines ${ }^{1}$ for recognition of death and termination of resuscitation attempts by ambulance service personnel in 1996 (fig l and 2). Shortly after the conclusion of this study, they published updated guidelines. The purpose of this survey was to see whether there was unanimity in the approach to the cardiac arrest victim by ambulance personnel from the various UK Ambulance trusts when compared with the nationally recommended guidelines in place at that time.

\section{METHODS}

Thirty nine ambulance trusts were identified using the "Directory of Emergency and Special Care Units". The training managers for each trust were contacted by post and asked what policies, if any, existed in their trust for "recognition of death" and termination of resuscitation attempts in the adult, normothermic, non-traumatic cardiorespiratory arrest victim. A follow up letter was sent three months later to those trusts that had not replied.

\section{RESULTS}

Thirty five trusts replied to the first letter and the remaining four trusts replied to the follow up letter. The results of the questionnaire are summarised in table 1.

- Seven trusts had no policy for termination of resuscitation attempts and would only allow their personnel to recognise death in the presence of irreversible factors (rigor mortis, postmortem staining, or injuries incompatible with life).

- Two trusts had a policy purely for termination of resuscitation.

- Two trusts had a policy purely for recognition of death in circumstances other than the presence of irreversible factors.

\section{GROUP A Conditions unequivocally associated with death}

- Decapitation

- Massive cranial and cerebral destruction

- Hemicorporectomy (or similar massive injury)

- Decomposition

- Incineration

- Rigor mortis

- Fetal maceration

In these groups, death can be recognised by the clinical confirmation of cardiac arrest

\section{GROUP B Conditions requiring ECG evidence of asystole}

- Submersion for more than three hours in adults over 18 years of age, with or without hypothermia

- Continuous asystole, despite cardiopulmonary resuscitation, for more than 20 minutes in a normothermic patient

- Patients who have recieved no resuscitation for at least 15 minutes after collapse and have no pulse or respiratory effort on arrival of the ambulance personnel

TIMINGS MUST BE ACCURATE

In all these cases, at least one minute's duration of continuous ECG record must be free from artifact and demonstrate asystole without QRS or $P$ waves. must be free from artifact and demonstrate asystole without QRS or P waves.
There must be no positive history of sedative, hypnotic, anxiolytic, opioid or There must be no positive history of sedative,
anaesthetic drugs in the preceding 24 hours.

\section{GROUP C Terminal illness}

- Cases of terminal illness where the doctor has given clear, written and signed instructions that the patient is not for resuscitation.

Figure 1 JRCALC guidelines 1996.

- Five trusts would only allow their personnel to withhold resuscitation attempts in the presence of irreversible factors but would allow them to terminate resuscitation attempts using protocols that recognise absence of bystander BLS but require them to deliver a limited period of CPR (see table 1).

- The remaining 23 trusts had individual policies for both circumstances.

The JRCALC recommended that the absence of BLS for 15 minutes (with or without one minute of CPR) and the absence 


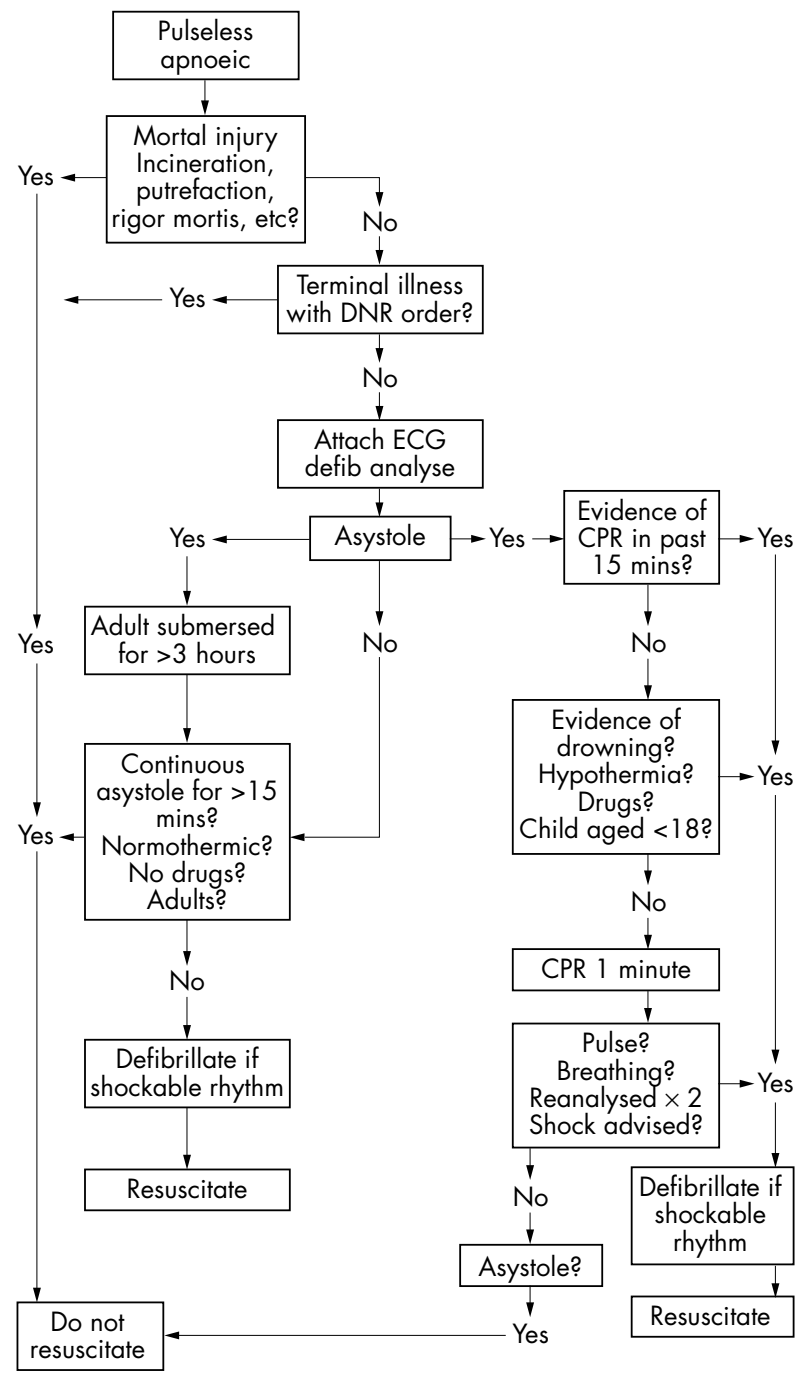

Figure 2 JRCLAC guidelines 1996.

of a response after 20 minutes of ALS were indications for "recognition of death", withholding or terminating resuscitation attempts. Only eight trusts followed these recommendations entirely.

\section{DISCUSSION}

The JRCALC proposed guidelines in $1996{ }^{1}$ for the "recognition of death". These outline explicit situations where the initiation of resuscitative measures would be inappropriate. They also made recommendations for when termination of ongoing resuscitation attempts may be appropriate (figs 1 and 2). The necessity to audit termination of resuscitation is now a statutory requirement for ambulance trusts.

There have been several guidelines proposed in the literature for the termination of resuscitative attempts on arrival of the patient at hospital. ${ }^{2-5}$ These all propose that the absence of a ventricular dysrhythmia along with adequate rescue attempts (intubation, defibrillation, IV medications, chest compression) are grounds for discontinuing active resuscitation. Eliastam ${ }^{2}$ states that there should have been no response after more than 30 minutes of ALS whereas Smith and Bodai ${ }^{5}$ propose that this should be extended to 45 minutes.

The issue of when to terminate resuscitative measures in the prehospital setting is also controversial. The International Guidelines $2000{ }^{6}$ suggest that, after all ALS interventions,

\section{Table 1}

Criteria

Number

Recognition of Death protocols

Rigor mortis/postmortem staining

No bystander BLS for 10 minutes + asystole

No bystander BLS for 15 minutes + asystole*

No bystander BLS for 20 minutes + asystole

13

Termination of Resuscitation protocols

No policy

ALS 10 minutes + persistent asystole

ALS 15 minutes + persistent asystole

ALS 20 minutes + persistent asystole*

"3 cycles" CPR + persistent asystole

14

Termination after limited period of CPR protocols

No bystander BLS for 10 minutes + CPR for 1

minute + persistent asystole

No bystander BLS for 15 minutes + CPR for 1

minute + persistent asystole*

No bystander BLS for 15 minutes + CPR for $2 \quad 2$

minutes + persistent asystole

No bystander BLS for 20 minutes + CPR for 3

minutes + persistent asystole

*Recommended by JRCALC 1996.

"continuously documented asystole $>5$ to 10 minutes" is a criteria for stopping resuscitative efforts in the out of hospital cardiac arrest.

There have been several other attempts to propose guidelines for the prehospital discontinuation of resuscitative attempts. ${ }^{7-12}$ It has been suggested that asystole and electromechanical dissociation are terminal rhythms and that these patients should not be transported to hospital. Bonnin ${ }^{7}$ proposes that in the absence of persistent ventricular fibrillation, resuscitation efforts can be terminated on scene in the normothermic adult who has had no return of spontaneous circulation within 25 minutes after standard advanced cardiac life support. In addition, Kellermann ${ }^{9}$ states "the burden of proof should shift to those who believe that rapid transport to the hospital is justified in cases of refractory out-of-hospital cardiac arrest."

More recently, the National Association of EMS Physicians ${ }^{13}$ have published eight factors that should be considered when terminating prehospital resuscitation. These include unwitnessed arrests with a delay to BLS beyond six minutes and a delay to defibrillation beyond eight minutes, and at least 20 minutes of ALS with no advanced directive.

In the UK, Marsden et al ${ }^{14}$ have proposed an algorithm for use by ambulance personnel faced with patients in whom attempts at resuscitation may be futile. They suggest that if there has been no evidence of CPR in the past 15 minutes and no evidence of a situation where survival has previously been seen after prolonged arrest (drowning, hypothermia, poisoning/overdose, children or pregnancy) then CPR is administered for one minute. This one minute of CPR is delivered in case there is a hypoxic origin to the cause of the arrest. After that, if there is no cardiac output and asystole persists then they advocate no further attempts at resuscitation. This approach allows a limited instigation of CPR to exclude hypoxic causes of cardiac arrest before subsequent termination of resuscitative attempts.

The JRCALC incorporated Marsden's algorithm into their recommendations as an alternative option. At the time of this study, the Scottish Ambulance Service, along with five other ambulance trusts, had adopted this protocol. In addition, there were three variants of this model being used by five other trusts. The reasoning why there are so many variations is unclear from this study, although possibilities may be because of training elements and/or communication issues within ambulance services. 


\section{CONCLUSION}

Based upon current evidence, the JRCALC proposed guidelines for "recognition of death" and terminating resuscitation attempts in the adult normothermic non-traumatic cardiac arrest. Despite this, there was still considerable variance in the practice of the UK ambulance trusts. Irrespective of any subsequent updated recommendations, this study shows that there was not a unified approach to managing this situation despite the presence of guidelines from an acknowledged body of experts. There should be unanimity in the approach by the UK ambulance trusts and further work needs to be done looking at adherence to the updated guidelines.

\section{ACKNOWLEDGEMENTS}

I would like to thank Professor D Chamberlain (Chairman of JRCALC) for his help and permission to reproduce the JRCALC recommendations.

Funding: none.

Conflicts of interest: none.

\section{REFERENCES}

1 Baskett P, Fisher J, Marsden A. Recognition of death by ambulance personnel. Joint Royal Colleges Ambulance Liaison Committee Newsletter 1996;1.

2 Eliastam M, Duralde T, Martinez F, et al. Cardiac arrest in the emergency medical service system: guidelines for resuscitation. Journal of American College of Emergency Physicians 1977;6:525-9.
3 Herlitz J, Ekstrom L, Axelsson A, et al. Continuation of CPR on admission to emergency department after out-of-hospital cardiac arrest. Occurrence, characteristics and outcome. Resuscitation 1997;33:223-31.

4 Van Hoeyweghen R, Mullie A, Bossaert L. Decision making to cease or to continue cardiopulmonary resuscitation (CPR). Resuscitation 1989;17 (suppl):S137-47.

5 Smith JP, Bodai BI. Guidelines for discontinuing prehospital CPR in the emergency department-a review. Ann Emerg Med 1985;14:1093-8.

6 AHA/ILCOR. International Guidelines 2000 for CPR and ECC-a consensus on science. Resuscitation 2000;46: 1-448.

7 Bonnin MJ, Pepe PE, Kimball KT, et al. Distinct criteria for termination of resuscitation in the out-of-hospital setting. JAMA 1993;270:1457-62.

8 Kellermann AL, Hackman BB, Somes G. Predicting the outcome of unsuccessful prehospital advanced cardiac life support. JAMA 1994;270: 1433-6.

9 Kellerman AL. Criteria for dead-on-arrivals, prehospital termination of CPR, and do-not-resuscitate orders. Ann Emerg Med 1993;22:47-5 1.

10 van der Hoeven JG, Waanders $\mathrm{H}$, Compier EA, et al. Prolonged resuscitation efforts for cardiac arrest patients who cannot be resuscitated at the scene: who is likely to benefit? Ann Emerg Med 1993;22:1659-63.

11 Faine PG, Willoughby PJ, Koenigsberg M, et al. Implementation of an out-of-hospital termination of resuscitation policy. Prehosp Emerg Care 1997; 1:246-52.

12 Aprahamian C, Thompson BM, Gruchow HW et al. Decision making in prehospital sudden cardiac arrest. Ann Emerg Med 1986;15:445-9.

13 Bailey ED, Wydro GC, Cone DC. Termination of resuscitation in the prehospital setting for adult patients suffering nontraumatic cardiac arrest. Prehosp Emerg Care 2000;4:190-5.

14 Marsden AK, Ng GA, Dalziel K, et al. When is it futile for ambulance personnel to initiate cardiopulmonary resuscitation? $B M$ 1995;311:49-51. 